\title{
Exploring Scalar NSI Effects in Long Baseline Neutrino Experiments
}

\author{
Abinash Medhi, ${ }^{1}$ Debajyoti Dutta, ${ }^{2}$ and Moon Moon Devi ${ }^{1}$ \\ ${ }^{1}$ Tezpur University, Napaam, Sonitpur, Assam-784 028, India \\ ${ }^{2}$ Assam Don Bosco University, Kamarkuchi, Sonapur, Assam-782 402, India
}

Abstract

Neutrino oscillations provide an excellent opportunity to look for new physics beyond the Standard Model or popularly known as BSM. The unknown couplings involving neutrinos, termed as Non Standard Interactions (NSIs) [1, 2], may appear as a 'new physics' in different neutrino experiments. The neutrino NSIs offer significant effects in neutrino oscillations and $\mathrm{CP}$ sensitivity, which can be probed in various neutrino experiments. The idea of scalar coupling term has evolved recently $[3,4]$ and looks promising. The effect of scalar NSI can appear as a perturbation that is added to the neutrino mass matrix in the neutrino Hamiltonian. As it modifies the neutrino mass matrix, it may provide a direct possibility of probing neutrino mass models. As the scalar NSI affects the neutrino mass matrix in the Hamiltonian, its effect is energy independent. Moreover, the matter effect due to scalar NSI scales linearly with matter density. In this work, we have performed a model independent study of the effects of scalar NSI at long baseline experiments (using DUNE [5]). Various neutrino parameters may get affected due to the inclusion of scalar NSI as it modifies the effective mass matrix of neutrinos. We explored the impact of scalar NSI in neutrino oscillations and its impact on measurement of various mixing parameters. We have studied different oscillation channels and explored the impact of various possible elements in the scalar NSI term.

Keywords: neutrino physics, non standard interactions, beyond standard model, scalar coupling, long baseline experiments DOI: 10.31526/ACP.BSM-2021.26

\section{INTRODUCTION}

The Discovery of neutrino oscillations [6, 7] essentially confirms neutrinos have non zero masses and Standard Model (SM) of elementary particles is not complete. It directly suggests that at least two of the three neutrinos should have masses [8]. Since the discovery of neutrino oscillations, numerous experiments are conducted and currently ongoing to determine various neutrino oscillation parameters precisely. Currently, the neutrino oscillation programs are entering into the precision era, where experiments are trying to find the known mixing angles and the mass square differences with utmost accuracy. The proposed next generation experiments are aimed to measure primarily three unknown oscillation parameters: Dirac $\mathrm{CP}$ phase $\left(\delta_{C P}\right)$ i.e. $\mathrm{CP}$ violation in leptonic sector, sign of atmospheric mass square differences $\left(\Delta m_{32}^{2}\right)$ i.e. neutrino mass ordering and octant of atmospheric mixing angle $\left(\theta_{23}\right)$ i.e. whether it lies in the lower octant $\left(\mathrm{LO}, \theta_{23}<45^{\circ}\right)$ or in the higher octant $\left(\mathrm{HO}, \theta_{23}>45^{\circ}\right)$. These analyses are generally performed within the framework of $3 \times 3$ neutrino mixing, considering neutrinos interact with matter only through $\mathrm{SM}$ weak interactions.

According to SM, neutrinos can interact with matter via weak interactions only, hence its interaction cross sections are very small as compared to other SM particles. When neutrinos pass through matter they interact via neutral current (NC) and charged current (CC) interactions, mediated by $\mathrm{Z}$ and $\mathrm{W}$ bosons respectively. In the design of a neutrino detector these neutrino interactions have to be taken into account, as the expected number of neutrinos in a detector is proportional to the cross section of the neutrino interactions. So understanding the interactions of neutrinos with the target of the detector is essential for correct detection of neutrinos in neutrino experiments. The neutrino matter effect played a crucial role in understanding the various phenomenology of neutrino oscillations.

The origin of neutrino mass clearly required an extension of SM to explain the mass and mixing of neutrinos. This gives a gateway to explore new physics beyond SM, which may get accompanied by some extra non standard interactions (NSIs) of neu- 
trinos with environmental matter fermions. Wolfenstein first pointed out the possibility of NSI in his pioneering paper [9], with generally parametrized vector and axial-vector currents, which was later explored by a large number of authors [reviews [1, 2, 10]]. In the paper [11] the authors discussed how one flavour changing NSI element $\left(\epsilon_{e \tau}\right)$ can affect the mass ordering sensitivity of $\mathrm{NO} v \mathrm{~A}$ and $\mathrm{T} 2 \mathrm{~K}$ significantly. This can completely washed out the current $\sim 2.4 \sigma$ indication of $\mathrm{NO} v \mathrm{~A}$ and $\mathrm{T} 2 \mathrm{~K}$ in favour of Normal Hierarchy (NH) of neutrino mass ordering. In the study [12] showed that the the discovery potential of octant of $\theta_{23}$ of DUNE gets completely lost in presence of off-diagonal $\left(\epsilon_{e \mu}\right.$ and $\left.\epsilon_{e \tau}\right)$ vector NSI elements. In presence of these NSI elements the transition probability $P_{\mu e}$ acquire an extra new interference term, which creates a degeneracy in measurement of $\delta_{C P}$. In another work [13] the author showed how diagonal and off-diagonal elements can severely effect the sensitivity of determination of $\delta_{C P}$ at DUNE. Not only DUNE, CP sensitivity of other long baseline (LBL) experiments like NOvA, T2K and proposed T2HK can also severely get affected by NSI elements [14]. The mass ordering sensitivities of different LBL experiments have been explored in this study [15]. For more on the studies of impact of scalar NSI elements on the sensitivity of LBL experiments are listed in these references $[16,17,18,19,20,21,22,23,24]$. A global status on the sensitivity of CP violation and neutrino mass ordering in presence of NSI in LBL experiments have been summarised in this study [25]. The study of NSI opens up the potentiality of using neutrino oscillations experiments to explore new physics scenarios beyond the SM. Such a new interaction leads to a rich phenomenology in both scattering experiments and neutrino oscillation experiments. Various experimental data are being analysed to see the bounds on such effects [26, 27].

The idea of NSI introduced by Wolfenstein in his paper [9] was vector-type interactions, where the neutrino interaction is mediated by a vector boson. The formulation of possible non standard couplings of neutrino to a scalar field was introduced recently [3]. This type of scalar interactions appear as a correction to the neutrino mass term and it can have a different phenomenological consequences than that of conventional NSI. Also, as the scalar NSI appears as an addition to the neutrino mass matrix, its impact on neutrino mass models are highly interesting and promising. These scalar couplings may also have an impact on the measurement of different neutrino oscillation parameters in various neutrino oscillation experiments. We make a model independent study of the effects of scalar NSI parameters on the long baseline neutrino experiments.

We organize the paper as follows: we discuss the formalism of scalar NSI in section 2. Then we present a brief a discussion on the probability in presence of scalar NSI in section 3. In section 4 we give a brief account on the methodology used in our study and discuss the results. We summarise our results in section 5 .

\section{SCALAR NSI FORMALISM}

According to SM, neutrinos can interact with matter via either SM charged current (CC) or neutral current (NC) interactions by mediating a $\mathrm{W}$ or $\mathrm{Z}$ bosons respectively. After Fierz transformation, [28, 29] these interactions can be written in the form $\left(\bar{v}_{\alpha} \gamma^{\mu} P_{L} v_{\alpha}\right)\left(\bar{f} \gamma_{\mu} P f\right)$ (with $f, f^{\prime} \in\{e, u, d\}$ the matter fermions and $P \in\left\{P_{L}, P_{R}\right\}$ the chirality projection operators. The chiral projection operators are given by $P_{L}=\left(1-\gamma_{5}\right) / 2$ and $\left.P_{R}=\left(1+\gamma_{5}\right) / 2\right)$. Then, the effective Lagrangian in presence of neutrino matter interactions (CC) can be written as :

$$
\mathcal{L}_{\mathrm{cc}}^{\mathrm{eff}}=-\frac{4 G_{F}}{\sqrt{2}}\left[\overline{v_{e}}\left(p_{3}\right) \gamma_{\mu} P_{L} v_{e}\left(p_{2}\right)\right]\left[\bar{e}\left(p_{1}\right) \gamma^{\mu} P_{L} e\left(p_{4}\right)\right]
$$

Where, $G_{F}$ is the Fermi constant. The matter effect comes from the forward scattering of neutrinos where zero momentum transfer happens i.e. $p_{1}=p_{4} \equiv p_{e}$ and $p_{2}=p_{3} \equiv p_{v}$. The matter potential can be calculated from the effective Lagrangian by sandwiching it with in and out states of the interactions. Then after some simplifications the matter potential gets into a simplified form [30] $V_{\mathrm{cc}}=-\bar{V}_{\mathrm{cc}}=\sqrt{2} G_{F} n_{e}$, where, $V_{\mathrm{cc}}$ is for neutrino case and $\bar{V}_{\mathrm{cc}}$ is for antineutrino case. $n_{e}$ is the number density of environmental electrons. So, the corresponding effective Hamiltonian for neutrino propagation in presence of neutrino matter effect is formalised as:

$$
\mathcal{H} \approx E_{v}+\frac{M M^{\dagger}}{2 E_{v}} \pm V_{\mathrm{SI}}
$$


where $\mathrm{M}$ is the neutrino mass matrix, $E_{v}$ is the neutrino energy and $V_{S I}$ is the matter potential induced by the standard neutrino interaction (SI) with matter. The ' + ' sign before $V_{S I}$ corresponds to neutrino and '-' sign is for antineutrino case.

A widely used way of incorporating NSI in neutrinos is by introducing a vector currents from a vector mediator or with Fierz transformation from a charged scalar [31,32] which leads to conventional vector-type NSI. The effect of this type of NSI is matter potential and its effect is studied extensively in the literature. However, neutrinos can also couple with a scalar field, particularly their non zero masses makes this possibility wide open. As, neutrinos can couple with a scalar (Higgs boson) with non zero vacuum expectation values to generate its mass. These type of non standard couplings of neutrinos are termed as scalar NSI.

The Lagrangian for scalar NSI induced by a neutral scalar mediator for Dirac neutrinos can be parametrize as :

$$
\mathcal{L}_{\text {eff }}^{S}=\frac{y_{f} y_{\alpha \beta}}{m_{\phi}^{2}}\left(\bar{v}_{\alpha}\left(p_{3}\right) v_{\beta}\left(p_{2}\right)\right)\left(\bar{f}\left(p_{1}\right) f\left(p_{4}\right)\right)
$$

Where, $\alpha, \beta=\mathrm{e}, \mu, \tau$ refer to the neutrino flavours, $\mathrm{f}=\mathrm{e}, \mathrm{u}, \mathrm{d}$ indicate the matter fermions, $y_{\alpha \beta}$ is the Yukawa couplings of the neutrinos with the scalar mediator, $\phi$ and $y_{f}$ is the Yukawa coupling of the mediator $\phi$ with the environmental fermions $f$. The $m_{\phi}$ is the mass of the scalar mediator $\phi$. Here, the Lagrangian is no longer composed of vector current as in the case of matter Lagrangian (Equation 1). Instead the Lagrangian is consisted of a scalar Yukawa term, which can't be converted to vector currents [33] and hence, it will not appear as a matter potential term. Rather, it appears as a medium-dependent correction to the neutrino mass term [3]. The effective Hamiltonian (Equation 3) for this kind of scalar interactions Lagrangian modifies as:

$$
\mathcal{H} \approx E_{v}+\frac{(M+\delta M)(M+\delta M)^{\dagger}}{2 E_{v}} \pm V_{\mathrm{SI}}
$$

Where, the expression for $\delta M$ is defined as $\quad \delta M \equiv \sum_{f} n_{f} y_{f} y_{\alpha \beta} / m_{\phi}^{2}$. Here $n_{f}$ is the number density of environmental fermion.

So, scalar NSI appears as an extra term added to the neutrino mass instead of appearing as a matter potential. Hence, the effect of scalar NSI is no longer matter potential and this makes the effect of scalar NSI quite different than that of the conventional NSI. Also, the scalar NSI effect is independent of the neutrino energy, whereas vector NSI scales with neutrino energy. Hence, the effect of scalar NSI is not suppressed at low energies. As $\delta M$ is proportional to matter density, scalar NSI can feel the matter density variation. All these can significantly effect the neutrino production, propagation and detection in various neutrino experiments. Also, as $\delta M$ is inversely proportional to mediator mass $m_{\phi}$, a light mediator is needed to see a large effect of scalar NSI in neutrino experiments. The study [4] has shown that, for any significant scalar NSI effect for neutrinos propagating in Earth, we need $G_{\text {eff }} \equiv y_{f} y_{\alpha \beta} / m_{\phi}^{2} \sim 10^{10} G_{F}$ with $\Delta m_{v} \sim \mathcal{O}\left(0.1 m_{v}\right)$. Anyway, scalar NSI can have a different phenomenological consequences in solar, reactor, atmospheric as well as accelerator neutrino oscillations.

We have used the following parametrization of $\delta M$ in our analysis to see the effects of scalar NSI in the neutrino experiment.

$$
\delta M \equiv \sqrt{\Delta m_{31}^{2}}\left(\begin{array}{lll}
\eta_{e e} & \eta_{e \mu} & \eta_{e \tau} \\
\eta_{\mu e} & \eta_{\mu \mu} & \eta_{\mu \tau} \\
\eta_{\tau e} & \eta_{\tau \mu} & \eta_{\tau \tau}
\end{array}\right)
$$

where $\eta_{\alpha \beta}$ are dimensionless parameters and it quantifies the size of the scalar NSI. The Hermicity of the neutrino Hamiltonian demands that the diagonal elements are real and off-diagonal elements are complex, which are parametrized as $\quad \eta_{\alpha \beta}=\left|\eta_{\alpha \beta}\right| e^{i \phi_{\alpha \beta}}$; 
for $\alpha \neq \beta$. This kind of formalism allow us to explore the impact of the elements in various probability channels. As $\eta$ elements quantify the size of the interactions, it can be tuned in the experiment to explain the results of various neutrino experiments. Currently, there are not any definite bounds on these elements, but it can be done by performing an analysis with different neutrino experiments.

In this work we have studied the effect of the scalar NSI elements $\left(\eta_{\alpha \beta}\right)$ at LBL experiments . We have developed a framework to study the effects of scalar NSI at LBL experiments, considering only the diagonal elements of mass correction matrix, $\delta M[E q u a t i o n$ 5]. We also performed the sensitivity analysis of different neutrino experiments towards finding these scalar NSI effects. On the other hand, the scalar NSI is not suppressed by either the non-relativistic environmental fermion or the relativistic neutrinos as, it directly affects the neutrino mass term, $M+\delta M$. As long as the correction due to scalar NSI, $\delta M$ and the neutrino mass term $M$ are comparable, the scalar NSI can have a significant effect in neutrino oscillations.

\section{OSCILLATION PROBABILITY IN PRESENCE OF SCALAR NSI}

Neutrino interactions have played a very important role in understanding the different properties of neutrinos. When neutrinos passes through a matter medium, a matter potential is added to the neutrino Hamiltonian called Wolfenstein matter potential. This matter potential due to the neutrino interactions with matter fermions, later affects the neutrino oscillation probabilities. The mixing angles of neutrinos can resonant to a maximal value for a particular value of matter density times the neutrino energy, called the MSW effect can significantly change the oscillation behaviour. Later this phenomenon was used to explain the solar neutrino flux of Sudbury Neutrino Observatory (SNO) data [7] and make a consistency with the terrestrial experiments [? ]. So, to understand any new interactions of neutrinos, it is necessary to understand its impact on the oscillation probabilities. Any new interactions added to the Lagrangian would eventually modify the Dirac equation of the neutrino propagation. Which will essentially affect the neutrino Hamiltonian hence the probabilities.

As the scalar NSI correction $(\delta M)$ is added to the mass term of the neutrino mass matrix, there is no essential difference between the genuine neutrino mass term $(\mathrm{M})$ and the correction added due to the scalar NSI. Even in the absence of genuine neutrino mass matrix neutrino oscillation still can happen due to the mass correction from scalar NSI. Fortunately, the the mass correction term depends on the matter density and genuine mass term is independent of environmental conditions. The mass correction due to scalar NSI is directly proportional to the matter density. The oscillation probability in LBL experiments can feel the matter density variations and by looking at the probabilities the effect of scalar NSI can be extracted.

For the LBL experiments like DUNE $[34,35,36,37,38]$, the most relevant probability channels are appearance channel $P_{\mu e}$ and the disappearance channel $P_{\mu \mu}$. For our study we have picked the diagonal elements of the scalar NSI mass correction matrix ( $\left.\delta M\right)$. We have taken one diagonal element of $\delta M$ at a time and explored its effect on the appearence probability $\left(v_{\mu} \rightarrow v_{e}\right)$ at the baseline of DUNE (1300 km).

\begin{tabular}{|c|c|}
\hline Parameters & True Values \\
\hline$\theta_{12}$ & $34.51^{\circ}$ \\
$\theta_{13}$ & $8.44^{\circ}$ \\
$\theta_{23}$ & $47^{\circ}$ \\
$\delta_{C P}$ & $-\pi / 2$ \\
$\Delta m_{21}^{2}$ & $7.56 \times 10^{-5}$ \\
$\Delta m_{31}^{2}$ & $2.43 \times 10^{-3}$ \\
\hline
\end{tabular}

TABLE 1: Oscillation parameters used in probability analysis

The mixing parameters values used in the analysis is listed in Table 1 . The values are in agreement with the latest global fit values provided by NuFIT 5.0 [39].

Figure 1 shows the appearance probability $P_{\mu e}$ vs Energy for DUNE. We have varied the energy of the neutrino beam from 0.5 to $10 \mathrm{GeV}$, which is the significant energy range for DUNE. The mixing parameters values taken in our analysis is listed in Table 1 


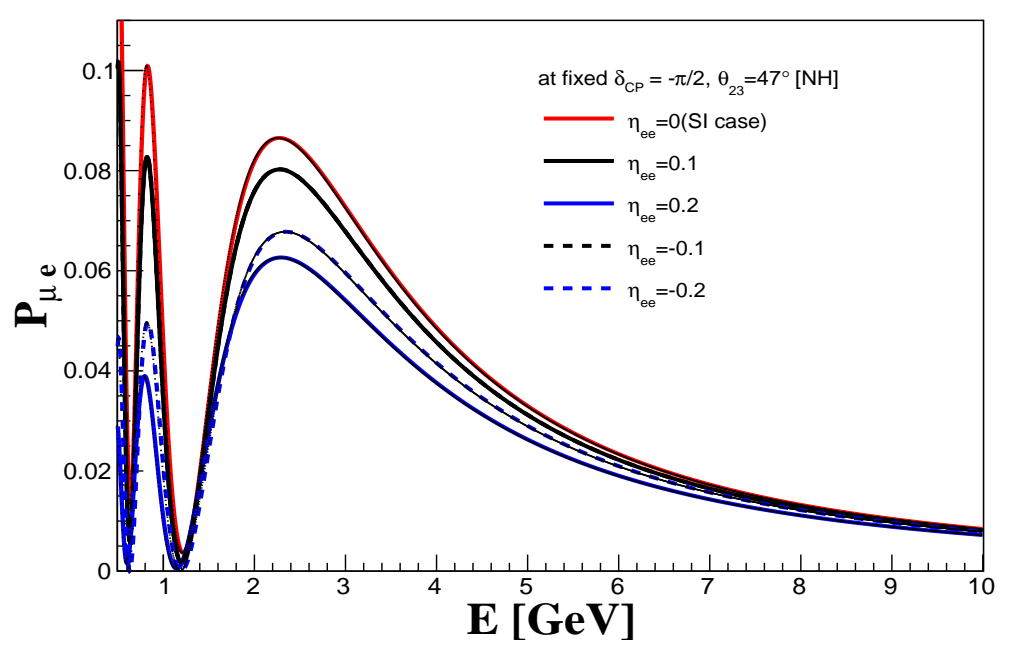

FIGURE 1: The effect of the diagonal scalar NSI element, $\eta_{e e}$ on neutrino appearance probabilities $\left(P_{\mu e}\right)$ at DUNE $($ baseline $=1300$ $\mathrm{km}$ ) for fixed $\delta_{C P}=-\pi / 2$ and $\theta_{23}=47^{\circ}$.

assuming normal hierarchy $[\mathrm{NH}]$. The plot shows the effect of diagonal scalar NSI element, $\eta_{e e}$ on the appearance probability $\left(P_{\mu e}\right)$ of DUNE for different values of $\eta_{e e} \in[-0.2,0.2]$. The solid red line shows the case of Standard Interaction (SI) with $\eta_{e e}=0$. The black and blue solid (dotted) curves are for non zero positive (negative) scalar NSI values. It can be seen that the effect of diagonal scalar NSI is quite significant on the probabilities of DUNE especially at the peaks. Similarly, the plot in the Figure 2 shows the effect of appearance probability $P_{\mu e}$ of DUNE in presence of diagonal $\eta_{\mu \mu}$ elements keeping all other NSI parameters as zeros. The red solid line corresponds to $\eta_{\mu \mu}=0$ i.e. matter SI case. We have varied $\eta_{\mu \mu}$ from -0.2 to 0.2 to check its effects on the probabilities. One interesting point to note here that the probability plots for positive and negative non zero $\eta_{\mu \mu}$ are symmetric around zero. For example the black solid and dashed plots corresponds to $\eta_{\mu \mu}=0.1$ and -0.1 respectively and both the probability plots are matching. So, for $\eta_{\mu \mu}$ case, only the absolute value of $\eta_{\mu \mu}$ is affecting the probabilities irrespective of its sign.

In Figure 3 and 4, oscillation probability $P_{\mu e}$ is checked with varying $\theta_{23}$ and $\delta_{C P}$ keeping $\eta_{e e}$ fixed at 0.01 and all other NSI elements as zeros respectively. The plots are for DUNE baseline (1300 km) and energy is in the range 0.5 to $10 \mathrm{GeV}$. The plots in Figure 3 represents the effect of $\theta_{23}$ on $P_{\mu e}$ in presence of scalar NSI. $\theta_{23}$ is varied within the allowed $3 \sigma$ range and $\delta_{C P}$ is fixed at $-\pi / 2$. It can be seen that with the increasing values of $\theta_{23}$ the peak of probabilities gets amplified i.e the effect of varying $\theta_{23}$ is at the peaks only. The plots in Figure 4 shows the effect of $\delta_{C P}$ on oscillation probabilities $P_{\mu e}$ for DUNE in presence of scalar NSI. Here also, The effect of varying $\delta_{C P}$ can be seen at the oscillation peaks. The values of $\delta_{C P}$ is varied in the range $-\pi$ to $+\pi$. The solid black plot corresponds to the no $\mathrm{CP}$ effect i.e. $\delta_{C P}=0$ and the solid (dashed) red lines are for the case of maximum $\mathrm{CP}$ effect i.e. $\delta_{C P}$ $=\pi / 2(-\pi / 2)$. The plot corresponding to $\delta_{C P}=\pi$ and $-\pi$ are matching with one another.

\section{RESULTS AND DISCUSSIONS}

\section{1. $\chi^{2}$ analysis Methodology}

In order to obtain the sensitivity of scalar NSI parameters we need to check whether an experiment can distinguish between non zero $\eta$ elements and SI case. To address this question we have used the following definition of $\chi^{2}$ to perform our experimental sensitivity analysis.

$$
\chi^{2} \equiv \min _{\eta} \sum_{i} \sum_{j} \frac{\left[N_{\text {true }}^{i, j}(\eta)-N_{\text {test }}^{i, j}(\eta)\right]^{2}}{N_{\text {true }}^{i, j}(\eta)}
$$




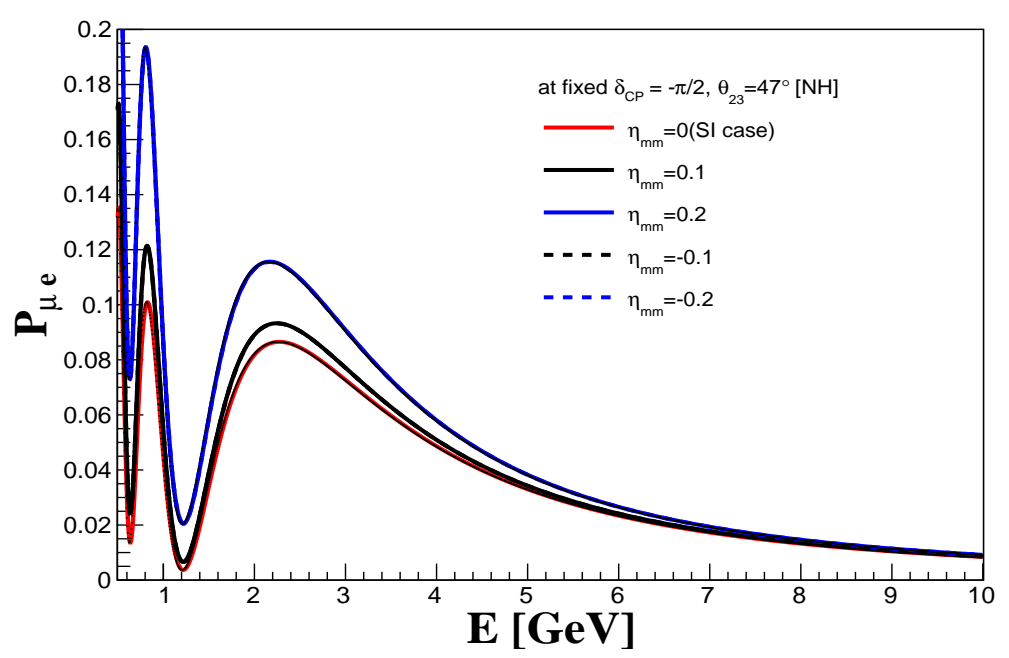

FIGURE 2: The effect of the diagonal scalar NSI elements $\eta_{\mu \mu}$ on neutrino appearance probabilities $\left(P_{\mu e}\right)$ at DUNE $($ baseline $=1300$ $\mathrm{km}$ ) for fixed $\delta_{C P}=-\pi / 2$ and $\theta_{23}=47^{\circ}$.

where $N_{\text {true }}^{i, j}$ and $N_{\text {test }}^{i, j}$ are the number of true and test events in the $\{i, j\}$-th bin respectively.

The package General Long baseline Experiment Simulator (GLoBES), [40, 41] has been used to perform our numerical simulations. The details of the experimental configurations used in the analysis are listed in Table 2.

The mixing parameters used in the analysis are given in the Table 1 . For the $\chi^{2}$ analysis, all the true values of the mixing parameters and scalar NSI elements are fixed. The true values of diagonal scalar NSI elements $\left(\eta_{e e}, \eta_{\mu \mu}\right.$ and $\left.\eta_{\tau \tau}\right)$ are set as 0.01 and all other off-diagonal values of $\eta$ elements and phases are taken as zeros. The test values of diagonal $\eta$ is marginalized in the range -0.15 to 0.15 for each $\chi^{2}$ analysis plots.

\begin{tabular}{|c|c|}
\hline Baseline & $1300 \mathrm{~km}$ \\
\hline Target Mass & 35 kton Liquid Argon TPC \\
\hline Run time & 5 years of $v$ mode and 5 years of $\bar{v}$ mode \\
\hline Energy Window & $0.375 \mathrm{GeV}-10.125 \mathrm{GeV}$ \\
\hline Profile type & single layer \\
\hline Matter density & $2.95 \mathrm{~g} / \mathrm{cm}^{3}$ \\
\hline
\end{tabular}

TABLE 2: Details of the detector configuration

\subsection{Analysis Results}

Assuming only the presence of a non-zero diagonal scalar NSI in nature, we have performed an analysis to check the sensitivity of DUNE towards these scalar NSI parameters. Figure 5 shows the $\chi^{2}$ sensitivity of DUNE towards the diagonal scalar NSI elements assuming normal hierarchy $(\mathrm{NH})$ as true hierarchy and for true $\theta_{23}=47^{\circ}(\mathrm{HO})$. The red solid line corresponds to true $\eta_{e e}=0.01$ and the black and blue dotted line correspond to true $\eta_{\mu \mu}=0.01$ and true $\eta_{\tau \tau}=0.01$ respectively. The plot for $\eta_{\mu \mu}$ and $\eta_{\tau \tau}$ is exactly same, only the effect of $\eta_{e e}$ it is different. The sensitivity of finding the $\eta_{e e}$ elements is little less for DUNE is as compared to the $\eta_{\mu \mu}$ and $\eta_{\tau \tau}$ elements.

The true values and marginalization values of parameters are listed in the following table.

Note that, the neutrino mass matrix is perturbed by the inclusion of scalar NSI, and it can effect the measurement of different parameters at long baseline experiments unconventionally. Also, as scalar NSI scales with matter density it can feel the matter density variation. According to this context, LBL experiments are an excellent candidate to see the such scalar NSI effects in neutrino oscillations. Because, in LBL experiments the neutrinos can feel the matter density variations as it travels a large distances. The appearance probabilities of DUNE shows that the diagonal scalar NSI parameters $\left(\eta_{e e}\right.$ and $\left.\eta_{\tau \tau}\right)$ can have a considerable impact on 


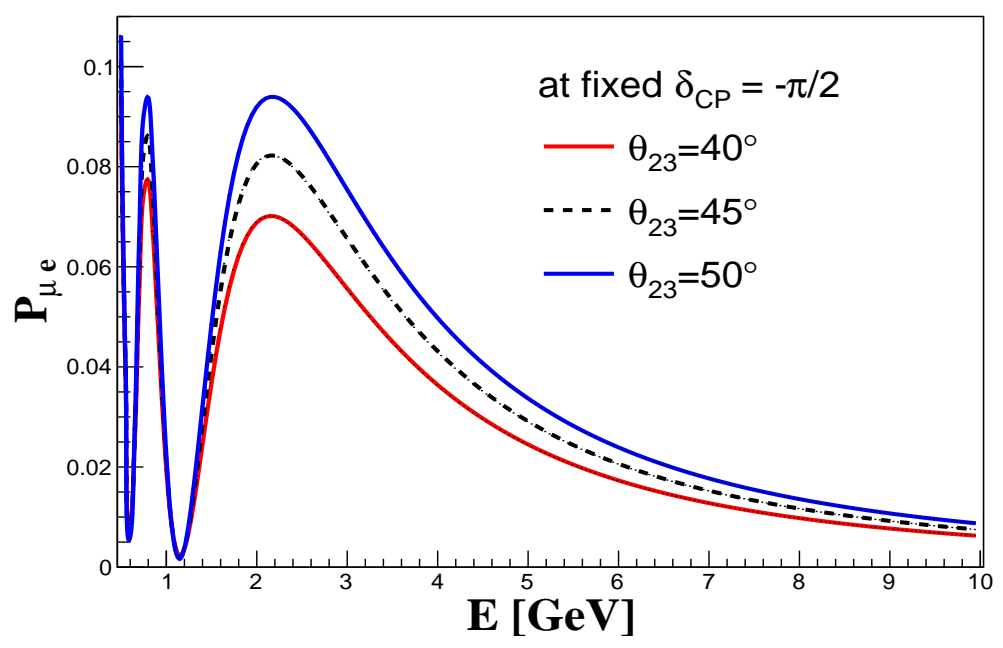

FIGURE 3: The effect of $\theta_{23}$ on neutrino oscillation probabilities $\left(P_{\mu e}\right)$ in presence of diagonal scalar NSI $\left(\eta_{e e}=0.01\right)$ at DUNE (baseline $=1300 \mathrm{~km}$ ) for fixed $\delta_{C P}=-\pi / 2$.

\begin{tabular}{|c|c|c|}
\hline Parameters & True Values & Marginalization \\
\hline$\theta_{12}$ & $34.51^{\circ}$ & FIXED \\
$\theta_{13}$ & $8.44^{\circ}$ & FIXED \\
$\theta_{23}$ & $47^{\circ}$ & FIXED \\
$\delta_{C P}$ & $-\pi / 2$ & FIXED \\
$\Delta m_{21}^{2}$ & $7.56 \times 10^{-5}$ & FIXED \\
$\Delta m_{31}^{2}$ & $2.43 \times 10^{-3}$ & FIXED \\
$\eta_{e e}$ & -0.15 to +0.15 & MARGINALIZED \\
$\eta_{\mu \mu}$ & -0.15 to +0.15 & MARGINALIZED \\
$\eta_{\tau \tau}$ & -0.15 to +0.15 & MARGINALIZED \\
$\eta_{e \mu}$ & 0 & FIXED \\
$\eta_{e \tau}$ & 0 & FIXED \\
$\eta_{\mu \tau}$ & 0 & FIXED \\
$\phi_{e \mu}$ & $-90^{\circ}$ & FIXED \\
$\phi_{e \tau}$ & $-90^{\circ}$ & FIXED \\
$\phi_{\mu \tau}$ & $-90^{\circ}$ & FIXED \\
\hline
\end{tabular}

the probabilities. Here, we have done our analysis considering only the diagonal elements of mass correction matrix $(\delta M)$. If the off-diagonal elements, $\eta_{\alpha \beta}(\alpha \neq \beta)$ and complex phases are also considered, these can make the situation more complicate and can be a trouble in the measurements of upcoming LBL experiments.

\section{SUMMARY AND CONCLUDING REMARKS}

In this work, we have explored the impact of scalar NSI parameters at the super-beam neutrino experiment DUNE. In particular, we have considered only the diagonal elements of scalar NSI parameters. We have seen that the effect of such diagonal scalar NSI elements have a notable impact on the oscillation probabilities. We have also performed a fixed $\chi^{2}$ analysis to see the effects of diagonal scalar NSI at DUNE. We have seen that $\chi^{2}$ sensitivity of $\eta_{\mu \mu}$ and $\eta_{\tau \tau}$ are same. Only, the $\eta_{e e}$ sensitivity is different than the others. The effect of scalar NSI can not be ignored in long baseline experiments. A global effort of different long baseline experiments is necessary to put some constrain on these scalar NSI parameters. Moreover, as scalar NSI affects the neutrino mass, there is also a possibility of probing it further into various neutrino mass models.

According to SM, neutrinos interact very weakly when it propagates through a medium. It interacts with matter via Charge current(CC) and/or Neutral current(NC) interactions with $\mathrm{W}$ and $\mathrm{Z}$ bosons as mediators of interactions respectively. Wolfenstein in his landmark paper[9] first pointed out that neutrinos can interact with matter. The neutrino matter effect played a crucial role in understanding the various phenomenology of neutrino oscillations. On the other hand, the origin of neutrino mass clearly required an extension of SM to explain the mass and mixing of neutrinos. This gives a gateway to new physics beyond SM, which often 


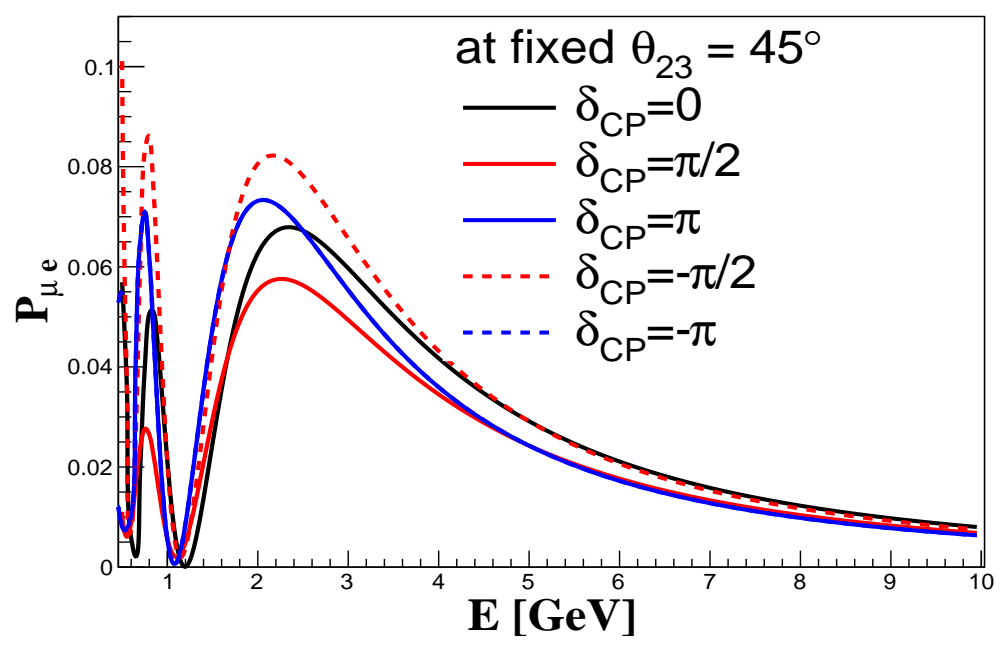

FIGURE 4: The effect of $\delta_{C P}$ on neutrino oscillation probabilities $\left(P_{\mu e}\right)$ in presence of diagonal scalar NSI $\left(\eta_{e e}=0.01\right)$ at DUNE (baseline $=1300 \mathrm{~km}$ ) for fixed $\delta_{C P}=-\pi / 2$.

comes with additional non standard interactions (NSIs) of neutrinos with matter fermions. Wolfenstein in his paper[9] introduced NSIs with generally parametrized vector and axial-vector currents. Since then, various studies [] has been done to understand the effects of NSI on the measurement of different neutrino oscillations parameters. By inclusion of these NSIs it can severely effect the neutrino production, propagation and detection. So it is therefore important to understand the effect of NSI and its size to interpret the experimental data in terms of relevant $3 \times 3$ oscillation parameters. The study of NSI also opens up the possibility of using neutrino oscillations to probe the origin of neutrino mass.

\section{ACKNOWLEDGEMENTS}

The work is supported by the Research and Innovation grant 2021, Tezpur University. MMD would like to acknowledge the support of DST SERB grant EMR/2017/001436 for this work.

\section{References}

[1] O. G. Miranda and H. Nunokawa. Non standard neutrino interactions: current status and future prospects. New J. Phys., 17(9):095002, 2015.

[2] Y. Farzan and M. Tortola. Neutrino oscillations and Non-Standard Interactions. Front. in Phys., 6:10, 2018.

[3] Shao-Feng Ge and Stephen J. Parke. Scalar Nonstandard Interactions in Neutrino Oscillation. Phys. Rev. Lett., 122(21):211801, 2019.

[4] K. S. Babu, Garv Chauhan, and P. S. Bhupal Dev. Neutrino nonstandard interactions via light scalars in the Earth, Sun, supernovae, and the early Universe. Phys. Rev. D, 101(9):095029, 2020.

[5] Babak Abi et al. Deep Underground Neutrino Experiment (DUNE), Far Detector Technical Design Report, Volume IV Far Detector Singlephase Technology. JINST, 15(08):T08010, 2020.

[6] Y. Fukuda et al. Evidence for oscillation of atmospheric neutrinos. Phys. Rev. Lett., 81:1562-1567, 1998.

[7] Q. R. Ahmad et al. Direct evidence for neutrino flavor transformation from neutral current interactions in the Sudbury Neutrino Observatory. Phys. Rev. Lett., 89:011301, 2002.

[8] M. Tanabashi et al. Review of Particle Physics. Phys. Rev. D, 98(3):030001, 2018.

[9] L. Wolfenstein. Neutrino Oscillations in Matter. Phys. Rev. D, 17:2369-2374, 1978.

[10] Carla Biggio, Mattias Blennow, and Enrique Fernandez-Martinez. General bounds on non-standard neutrino interactions. JHEP, 08:090, 2009.

[11] Francesco Capozzi, Sabya Sachi Chatterjee, and Antonio Palazzo. Neutrino Mass Ordering Obscured by Nonstandard Interactions. Phys. Rev. Lett., 124(11):111801, 2020.

[12] Sanjib Kumar Agarwalla, Sabya Sachi Chatterjee, and Antonio Palazzo. Degeneracy between $\theta_{23}$ octant and neutrino non-standard interactions at DUNE. Phys. Lett. B, 762:64-71, 2016.

[13] K. N. Deepthi, Srubabati Goswami, and Newton Nath. Challenges posed by non-standard neutrino interactions in the determination of $\delta_{C P}$ at DUNE. Nucl. Phys. B, 936:91-105, 2018. 


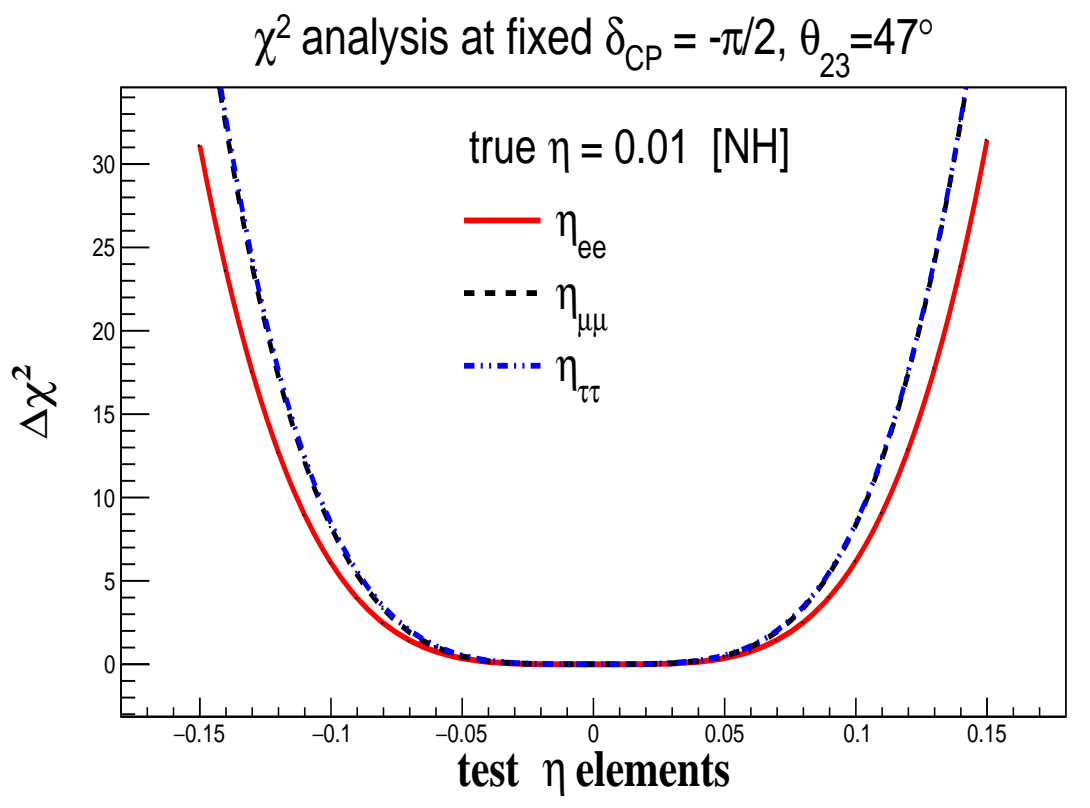

FIGURE 5: Fixed $\chi^{2}$ analysis for DUNE in presence of diagonal scalar NSI elements for true $\delta_{C P}=-\pi / 2$ and true $\theta_{23}=47^{\circ}$.

[14] Mehedi Masud and Poonam Mehta. Nonstandard interactions spoiling the $c p$ violation sensitivity at dune and other long baseline experiments. Phys. Rev. D, 94:013014, Jul 2016.

[15] Mehedi Masud and Poonam Mehta. Nonstandard interactions and resolving the ordering of neutrino masses at DUNE and other long baseline experiments. Phys. Rev. D, 94(5):053007, 2016.

[16] Alexander Friedland and Ian M. Shoemaker. Searching for Novel Neutrino Interactions at NOvA and Beyond in Light of Large $\theta_{13} .72012$.

[17] Joao A. B. Coelho, Tomas Kafka, W. Anthony Mann, Jacob Schneps, and Ozgur Altinok. Constraints for non-standard interaction $\epsilon_{e \tau} V_{e}$ from $v_{e}$ appearance in MINOS and T2K. Phys. Rev. D, 86:113015, 2012.

[18] Zini Rahman, Arnab Dasgupta, and Rathin Adhikari. The Discovery reach of $C P$ violation in neutrino oscillation with non-standard interaction effects. J. Phys. G, 42:065001, 2015.

[19] Pilar Coloma. Non-Standard Interactions in propagation at the Deep Underground Neutrino Experiment. JHEP, $03: 016,2016$.

[20] André de Gouvêa and Kevin J. Kelly. Non-standard Neutrino Interactions at DUNE. Nucl. Phys. B, 908:318-335, 2016.

[21] Jiajun Liao, Danny Marfatia, and Kerry Whisnant. Degeneracies in long-baseline neutrino experiments from nonstandard interactions. Phys. Rev. D, 93(9):093016, 2016.

[22] David V. Forero and Patrick Huber. Hints for leptonic CP violation or New Physics? Phys. Rev. Lett., 117(3):031801, 2016.

[23] Katri Huitu, Timo J. Kärkkäinen, Jukka Maalampi, and Sampsa Vihonen. Constraining the nonstandard interaction parameters in long baseline neutrino experiments. Phys. Rev. D, 93(5):053016, 2016.

[24] Pouya Bakhti and Yasaman Farzan. CP-Violation and Non-Standard Interactions at the MOMENT. JHEP, 07:109, 2016.

[25] Ivan Esteban, M. C. Gonzalez-Garcia, and Michele Maltoni. On the Determination of Leptonic CP Violation and Neutrino Mass Ordering in Presence of Non-Standard Interactions: Present Status. JHEP, 06:055, 2019.

[26] Sandhya Choubey, Anushree Ghosh, Tommy Ohlsson, and Deepak Tiwari. Neutrino Physics with Non-Standard Interactions at INO. JHEP, 12:126, 2015.

[27] Amina Khatun, Sabya Sachi Chatterjee, Tarak Thakore, and Sanjib Kumar Agarwalla. Enhancing sensitivity to non-standard neutrino interactions at INO combining muon and hadron information. Eur. Phys. J. C, 80(6):533, 2020.

[28] Markus Fierz. Zur fermischen theorie des $\beta$-zerfalls. Zeitschrift für Physik, 104(7):553-565, 1937.

[29] Jose F. Nieves and Palash B. Pal. Generalized Fierz identities. Am. J. Phys., 72:1100-1108, 2004.

[30] Rabindra N Mohapatra and Palash B Pal. Massive neutrinos in physics and astrophysics, volume 72. World scientific, 2004.

[31] Michal Malinsky, Tommy Ohlsson, and He Zhang. Non-Standard Neutrino Interactions from a Triplet Seesaw Model. Phys. Rev. D, 79:011301, 2009.

[32] M. B. Gavela, D. Hernandez, T. Ota, and W. Winter. Large gauge invariant non-standard neutrino interactions. Phys. Rev. D, 79:013007, 2009.

[33] C. C. Nishi. Simple derivation of general Fierz-like identities. Am. J. Phys., 73:1160-1163, 2005.

[34] William Marciano and Zohreh Parsa. Intense neutrino beams and leptonic CP violation. Nucl. Phys. B Proc. Suppl., 221:166-172, 2011.

[35] M. Bass et al. Baseline Optimization for the Measurement of CP Violation, Mass Hierarchy, and $\theta_{23}$ Octant in a Long-Baseline Neutrino Oscillation Experiment. Phys. Rev. D, 91(5):052015, 2015.

[36] R. Acciarri et al. Long-Baseline Neutrino Facility (LBNF) and Deep Underground Neutrino Experiment (DUNE): Conceptual Design Report, Volume 2: The Physics Program for DUNE at LBNF. 122015. 
[37] R. Acciarri et al. Long-Baseline Neutrino Facility (LBNF) and Deep Underground Neutrino Experiment (DUNE): Conceptual Design Report, Volume 4 The DUNE Detectors at LBNF. 12016.

[38] R. Acciarri et al. Long-Baseline Neutrino Facility (LBNF) and Deep Underground Neutrino Experiment (DUNE): Conceptual Design Report, Volume 1: The LBNF and DUNE Projects. 12016.

[39] Ivan Esteban, M. C. Gonzalez-Garcia, Michele Maltoni, Thomas Schwetz, and Albert Zhou. The fate of hints: updated global analysis of three-flavor neutrino oscillations. JHEP, 09:178, 2020.

[40] Patrick Huber, M. Lindner, and W. Winter. Simulation of long-baseline neutrino oscillation experiments with GLoBES (General Long Baseline Experiment Simulator). Comput. Phys. Commun., 167:195, 2005.

[41] Patrick Huber, Joachim Kopp, Manfred Lindner, Mark Rolinec, and Walter Winter. New features in the simulation of neutrino oscillation experiments with GLoBES 3.0: General Long Baseline Experiment Simulator. Comput. Phys. Commun., 177:432-438, 2007. 